\title{
A Novel Double-Loop Vector Control Strategy for PMSMs Based on Kinetic Energy Feedback
}

\author{
Anbang Wang ${ }^{\dagger}$, Qunjing Wang,**, and Weidong Jiang* \\ ${ }^{\dagger, *}$ School of Electrical Engineering and Automation, Hefei University of Technology, Hefei, China \\ ${ }^{* *}$ School of Electrical Engineering and Automation, Anhui University, Hefei, China
}

\begin{abstract}
A novel vector control strategy for a permanent magnet synchronous motor (PMSM) based on the kinetic energy stored in the rotor is proposed in this paper. The novel strategy is composed of two closed loops, in which the current loop is the inner loop, and the kinetic energy serves as the outer loop. The theoretical basis and the design procedure of the two loops are given. The feasibility of the proposed control strategy is verified by experimental results. When compared with traditional vector control strategies, the proposed vector control strategy based on energy feedback has better dynamic performance. In addition, an effective estimation solution for the load variation is put forward.
\end{abstract}

Key words: Kinetic energy feedback, Load estimation, PMSM, Vector control

\section{INTRODUCTION}

Permanent magnet synchronous motors (PMSMs) possess the advantages of high power density, high torque-to-inertia ratio, and high efficiency. They have been widely used in many industrial applications, such as tractions, robotics, and spindle drives. Due to the features of multi-variables, non-linearity and strong coupling, a sufficiently high control performance for this kind of system is difficult to obtain.

With the development of motor control techniques and modern control theory, many control methods have been applied to PMSM systems to improve their performance. Noguchi $\mathrm{T}$ firstly proposed the direct power control (DPC) [1] method, in 1997. Since then, DPC has been used for permanent magnet synchronous motors [2], by which the optimum voltage vector is selected to control the instantaneous power through an optimum switching table, based on the sector of the flux linkage and the output of the active and reactive power hysteresis. This method shows the good performance of the control system. Predictive control [3], fractional order control [4], sliding mode control [5], finite-time control [6], [7], robust control [8], disturbance

Manuscript received Nov. 6, 2014; accepted Apr. 11, 2015

Recommended for publication by Associate Editor Kwang-Woon Lee.

${ }^{\dagger}$ Corresponding Author: delsuner@163.com

Tel: +86-551-6588-3869, Hefei University of Technology

*School of Electrical Eng. and Automation, Hefei Univ. of Tech., China

${ }^{* *}$ School of Electrical Eng. and Automation, Anhui University, China rejection control [9], intelligent control [10], [11], and adaptive control [12]-[15] have also been applied to PMSM systems.

Generally, the kinetic energy of a motor is a square function of the rotor's speed. This means that the energy storage increases as a quadratic function of the speed. From the perspective of energy, a novel vector control strategy is proposed in this paper. The difference between the reference value and the actual value of the kinetic energy is the input of the outer loop in the proposed control strategy. In addition, the output of the outer loop is the reference value of the power. By decoupling the induced electromotive force, the reference value of the current can be acquired as the input of inner loop. In order to verify the dynamic performance of the vector control strategy based on the energy feedback, this strategy is compared with the traditional vector control (VC) strategy. At last, the influence of the rotational inertia and the load torque variation of the proposed control strategy is studied, and an effective load estimation solution is put forward in this paper to solve the instability of the PMSM control system.

Kinetic energy is chosen as the control variable in some papers [16] concerning flywheel energy storage applications. Without the load estimation method and the feed-forward control, this control strategy is unsuitable for systems with a variable inertia. Consequently, the proposed control strategy in this paper enjoys a broader applicability. 


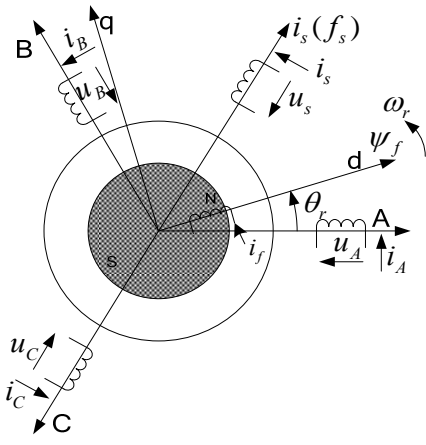

Fig. 1. Voltage vector and current vector.

\section{MAthematical Models OF A PMSM BASED ON ENERGY FEEDBACK}

\section{A. Voltage Equations of a PMSM}

In the $\mathrm{d}-\mathrm{q}$ axis coordinates, the PMSM voltage equation can be expressed as:

$$
\left\{\begin{array}{l}
U_{d}=R_{s} i_{d}+L_{d} \frac{d i_{d}}{d t}-\omega_{s} L_{q} i_{q} \\
U_{q}=R_{s} i_{q}+L_{q} \frac{d i_{q}}{d t}+\omega_{s}\left(L_{d} i_{d}+\Psi_{f}\right)
\end{array}\right.
$$

where $L_{d}$ and $L_{q}$ are the $d$-axis and $q$-axis synchronous inductances, respectively, $\omega_{s}$ is the angle frequency of the power source, and $\Psi_{f}$ is the excitation flux of the PMSM. In the steady-state, $i_{d}$ and $i_{q}$ are constant. Substituting the counter electromotive force $e_{0}=\omega_{s} \Psi_{f}$ into Eq. (1) yields:

$$
\left\{\begin{array}{c}
U_{d}=R_{s} i_{d}-\omega_{s} L_{q} i_{q} \\
U_{q}=R_{s} i_{q}+\omega_{s} L_{d} i_{d}+e_{0}
\end{array}\right.
$$

\section{B. Mathematical Models of a PMSM Based on Energy Feedback}

In the static three-phase coordinates, $\boldsymbol{U}_{\boldsymbol{A B C}}$ and $\boldsymbol{I}_{\boldsymbol{A B C}}$ are set as the phase voltage vector and the current vector, respectively. Then, the corresponding instantaneous values are expressed as:

$$
\left\{\begin{array}{l}
\boldsymbol{U}_{A B C}=\left[\begin{array}{lll}
u_{A} & u_{B} & u_{C}
\end{array}\right] \\
\boldsymbol{I}_{A B C}=\left[\begin{array}{lll}
i_{A} & i_{B} & i_{C}
\end{array}\right]
\end{array}\right.
$$

The voltage vector and the current vector in the static three-phase coordinates are shown in Fig. 1. The instantaneous active power $P$ is the scalar product of $\boldsymbol{U}_{A B C}$ and $\boldsymbol{I}_{\boldsymbol{A B C}}$, while the instantaneous reactive power $Q$ is the vector product of $\boldsymbol{U}_{A B C}$ and $\boldsymbol{I}_{A B C}$. In the static three-phase coordinates, the expression for $P$ and $Q$ is:

$$
\left[\begin{array}{l}
P \\
Q
\end{array}\right]=\left(\begin{array}{ccc}
u_{A} & u_{B} & u_{C} \\
u_{A}^{*} & u_{B}^{*} & u_{C}^{*}
\end{array}\right)\left[\begin{array}{c}
i_{A} \\
i_{B} \\
i_{C}
\end{array}\right]
$$

where $u_{A}{ }^{*}, u_{B}{ }^{*}$, and $u_{C}{ }^{*}$ are the conjugates of $u_{A}, u_{B}$, and $u_{C}$, respectively. When converting Eq. (4) into the rotary two-phase coordinates, the equations are given as:

$$
\left[\begin{array}{l}
P \\
Q
\end{array}\right]=\left(\begin{array}{cc}
u_{d} & u_{q} \\
u_{q} & -u_{d}
\end{array}\right)\left[\begin{array}{l}
i_{d} \\
i_{q}
\end{array}\right]
$$

Ignoring the eddy-current loss and the magnetic-hysteresis loss, the power input of the PMSM by the inverter has two main parts. The first one is to balance the load; the second one is the variation of the kinetic energy stored in the motor. Then the following equation can be obtained:

$$
\frac{1}{2} J \frac{d \omega_{s}^{2}}{d t}+T_{L} \omega_{s}=u_{d} i_{d}+u_{q} i_{q}
$$

In order to simplify the analysis, considering a motor with a constant torque load, the load torque $T_{L}$ is ignored in the dynamic process. By ignoring the load torque, Eq. (6) can be simplified as:

$$
P=\frac{1}{2} J \frac{d \omega_{s}^{2}}{d t}=\frac{1}{2} \times \frac{J}{9.55^{2}} \times \frac{d n^{2}}{d t}
$$

The equation based on the voltage equation of the PMSM is expressed as:

$$
u_{q}=\sqrt{\frac{3}{2}} K_{E} \frac{n}{1000}
$$

where $K_{E}$ denotes the voltage constant. In order to realize the maximum ratio of torque to current for surface-mounted PMSM control systems, the reference value of the current of the $\mathrm{d}$ axes $i_{d}{ }^{*}$ is set to zero. Combined with Eq. (5), the equation is expressed as:

$$
P^{*}=u_{q} i_{q}+u_{d} i_{d}=R_{s} i_{q}{ }^{2}+\frac{1}{2} L_{q} \frac{d i_{q}{ }^{2}}{d t}+e_{0} i_{q}
$$

From Eq. (9), the reference values $i_{d}{ }^{*}$ and $i_{q}{ }^{*}$ of the $\mathrm{d}$ and q axes are expressed as:

$$
\left\{\begin{array}{l}
i_{d}^{*}=0 \\
i_{q}^{*}=\frac{P^{*}}{u_{q}}=\frac{1000 \sqrt{2} P^{*}}{\sqrt{3} K_{E} n}
\end{array}\right.
$$

Ignoring the power loss of $R_{s}$ and the energy stored in $L_{q}, u_{q}$ can be substituted by $e_{0}$.

\section{DESIGN OF THE PMSM VECTOR CONTROL SYSTEM}

\section{A. Control Block Diagrams of the Two Strategies}

As shown in Fig. 2 (a), the traditional vector control for PMSMs adopts a two-closed-loop control, which is comprised of an outer speed loop and an inner current loop. In order to reduce the adjustment range of the inner loop, the induction electromotive force should be fed forward. 


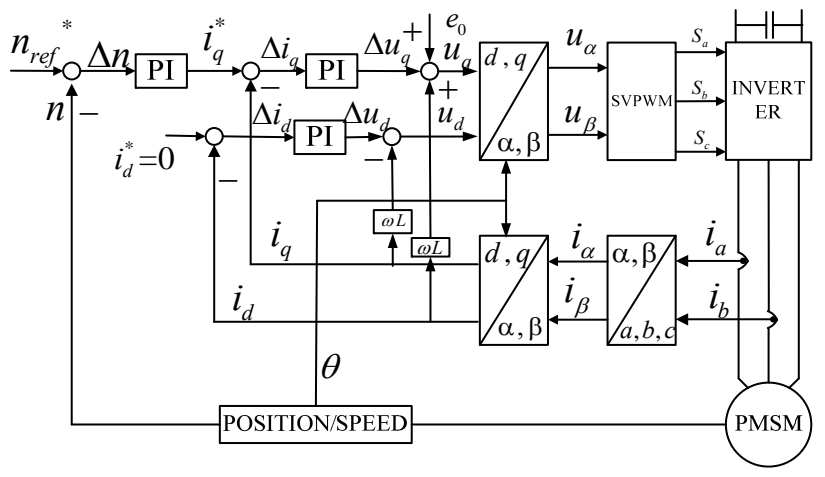

(a) Traditional vector control block diagram.

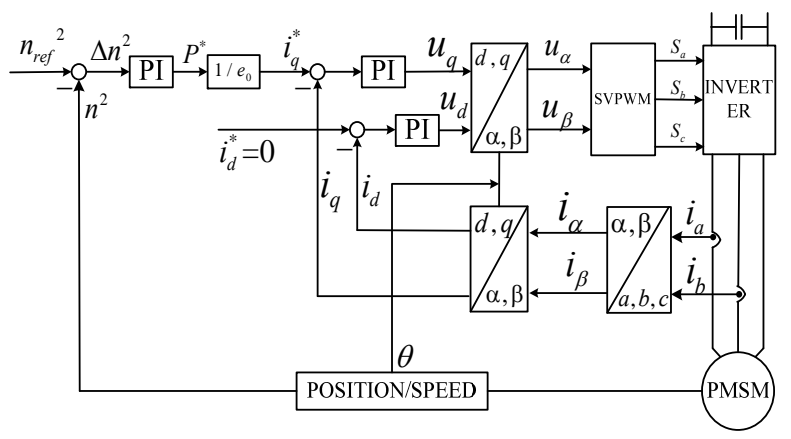

(b) Control block diagram based on energy feedback.

Fig. 2. Control block diagram of the two strategies.

The input of the kinetic energy PI controller is the difference between the reference value and the actual value of the kinetic energy of the motor. In addition, the output of the kinetic energy PI controller is the reference value of the active power. When this is divided by the induction electromotive force, $i_{q}^{*}$ can be acquired.

In order to facilitate understanding, the speed direction is ignored. Then, in the actual situations, the square items of the speed $\mathrm{n}_{r e f}{ }^{2}$ and $\mathrm{n}^{2}$ are substituted with $\mathrm{n}_{r e f} \cdot\left|\mathrm{n}_{r e f}\right|$ and $\mathrm{n} \cdot|\mathrm{n}|$, respectively.

\section{B. Design of the Current Loop}

The current loop of the PMSM vector control based on energy feedback is designed according to the traditional design method. Fig. 3 demonstrates the design of the current loop.

In Fig. 3, $T_{s}$ is the current sampling period. $K_{i P}$ and $K_{i I}$ are the proportional coefficient and integral coefficient of the current loop, respectively. $K_{P W M}$ is the PWM equivalent gain of the bridge circuit, $R$ is the phase resistance of the motor stator, and $T_{l}$ is the electrical time constant of the motor. Ignoring the dynamic influence of the induced electromotive force, the current PI controller is expressed in the zero-pole form:

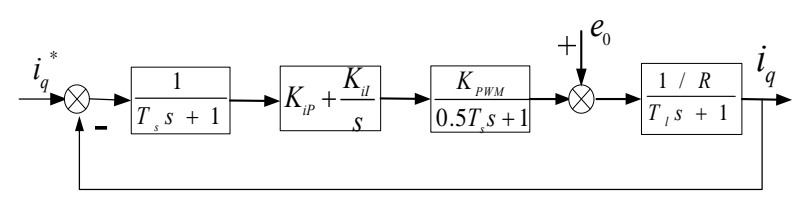

(a) The q axis current loop block diagram.

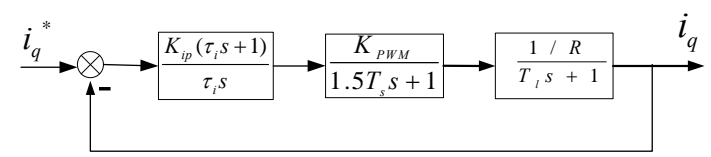

(b) The simplified q axis current loop block diagram.

Fig. 3. The design of the current loop.

$$
K_{i P}+\frac{K_{i I}}{\tau_{i} s}=\frac{K_{i P}\left(\tau_{i} s+1\right)}{\tau_{i} s}, K_{i I}=\frac{K_{i P}}{\tau_{i}}
$$

Ignoring the square item of S, Fig. 3(a) is simplified as Fig. 3(b).

Considering that the current loop needs faster tracking performance, the current PI controller should be designed according to a first order system, and the large time constant should be counteracted by the zero of the PI controller, that is $\tau_{i}=T_{l}$. Thus, the open-loop transfer function of the inner current loop is given as the following equation:

$$
W_{o p i}=\frac{K_{i P} K_{P W M}}{R \tau_{i} s\left(1.5 T_{s} s+1\right)}
$$

Hence, the closed-loop transfer function of the inner current loop is:

$$
W_{c l i}=\frac{1}{1+\frac{\tau_{i} R}{K_{i P} K_{P W M}} s+\frac{1.5 T_{s} \tau_{i} R}{K_{i P} K_{P W M}} s^{2}}
$$

If there is no special requirement, the damping ratio value of the system is: $\xi=\frac{\sqrt{2}}{2}$, and it is easy to obtain:

$$
\tau_{i}=\frac{3 T_{s} K_{i p} K_{P W M}}{R}
$$

Then the proportional coefficient and integral coefficient of the current loop are expressed by the following equations:

$$
\left\{\begin{array}{l}
K_{i P}=\frac{R \tau_{i}}{3 T_{s} K_{P W M}} \\
K_{i I}=\frac{K_{i p}}{\tau_{i}}=\frac{R}{3 T_{s} K_{P W M}}
\end{array}\right.
$$

When the switching frequency is high enough, that is, when $T_{s}$ is sufficiently small, the higher-order terms of the system can be ignored. When Eq. (15) is substituted into Eq. (13), Eq. (12) is simplified as:

$$
W_{c l i} \approx \frac{1}{1+3 T_{s} s}
$$




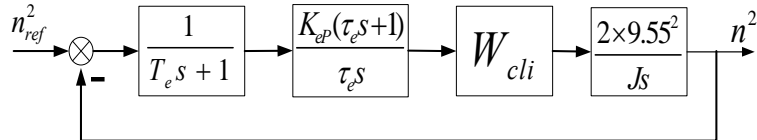

(a) The block diagram of the kinetic energy loop.

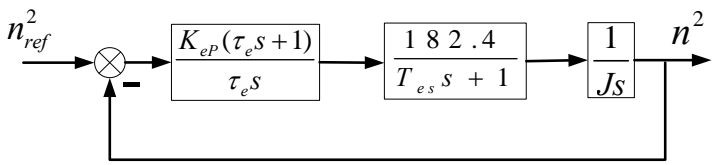

(b) The simplified block diagram of the kinetic energy loop.

Fig. 4. The design of the kinetic energy loop.

\section{Design of the Kinetic Energy Loop}

Tracking performance is the most important factor in the current loop. When designing the kinetic energy loop, the inner loop can be equivalent to a first order inertia link. A block diagram of the kinetic energy loop is obtained and shown in Fig. 4.

In Fig. 4, $T_{e}$ is the delay period of the kinetic energy loop, $W_{c l i}$ is the closed-loop transfer function of the current loop (see Eq. (16)), $J$ is the rotational inertia of the motor and the load, $K_{e P}$ is the proportional coefficient of the kinetic energy loop, and $K_{e I}$ is the integral coefficient of the kinetic energy loop. Thus, the zero-pole form of the kinetic energy loop is:

$$
K_{e P}+\frac{K_{e I}}{\tau_{e} s}=\frac{K_{e P}\left(\tau_{e} s+1\right)}{\tau_{e} s}, \quad K_{e l}=\frac{K_{e P}}{\tau_{e}}
$$

The current equivalent small time constant of the inner loop and the small inertia link delay of the kinetic energy loop should be combined; that is, $T_{e s}=T_{e}+3 T_{s}$. Therefore, Fig. 4(b) is obtained.

In terms of energy, the kinetic energy loop controls the speed of the motor. In the design of the kinetic energy loop, more attention should be paid to the anti-interference performance. The controller is designed according to a second order system. The open-loop transfer function of the kinetic energy loop from Fig. 4(b) is obtained as:

$$
W_{\text {ope }}=\frac{182.4 K_{e P}\left(\tau_{e} s+1\right)}{J \tau_{e} s^{2}\left(T_{e s} s+1\right)}
$$

The bandwidth of the kinetic energy loop is defined as:

$$
h=\frac{\tau_{e}}{T_{e s}}
$$

According to the parameter-tuning rule of a second order system controller, the equation is:

$$
\frac{182.4 K_{e P}}{J \tau_{e}}=\frac{h+1}{2 h^{2} T_{e s}^{2}}
$$

Under normal circumstances, the amount of the overshoot is reduced with an increase of h. However, the adjustment time is not monotonic with a change of h. If the tracking and anti-interference performance of the second order system are both taken into consideration, the value of $h$ is normally selected as 5 . The proportional and integral coefficients of the PI controller of the kinetic energy loop are expressed as:

$$
\left\{\begin{array}{l}
\tau_{e}=5 T_{e s}=5\left(T_{e}+3 T_{s}\right) \\
K_{e P}=\frac{J}{304\left(T_{e}+3 T_{s}\right)} \\
K_{e I}=\frac{K_{e P}}{\tau_{e}}
\end{array}\right.
$$

\section{Analysis of the Load Variation and the Load Compensation}

The kinetic energy stored in the rotor is related to the rotational inertia of the motor. By ignoring the friction of system, the actual exchange relationship of the energy between the motor and the drive system is:

$$
p_{D}=p_{A}+R_{s} i_{q}^{2}+\frac{1}{2} L_{q} \frac{d i_{q}^{2}}{d t}+\frac{1}{2 p_{0}^{2}} J_{L} \frac{d \omega_{s}^{2}}{d t}+p_{L}
$$

where $p_{D}$ is the total input electric power of the inverter, $J$ and $J_{\mathrm{L}}$ are the rotational inertia values of the rotor and the load machine, respectively, and $p_{A L}=\frac{1}{2 p_{0}^{2}} J_{L} \frac{d \omega_{s}^{2}}{d t}$ is the required power for the change of the load speed. When the load speed is changed, the item is present; when the load operates at a stable speed, it is zero. $p_{A L}+p_{L}$ is considered as one item of $p_{L}^{\prime}$.

When the load is changed, there is no longer balance between the power of the AC side and the load power. Due to the lag of the PI regulator, the load should exchange energy with the kinetic energy of the rotor first. According to the power equation, and ignoring the power loss of $R_{s}$ and the energy stored in $L_{q}$, the feed-forward estimation for the load power can be written as:

$$
p_{L}^{\prime *}=\frac{\sum\left(e_{q} i_{q}\right) \Delta T}{k \Delta T}-\frac{\Delta\left(\frac{1}{2} J \frac{\omega_{s}^{2}}{p_{0}{ }^{2}}\right)}{k \Delta T}
$$

In (23), the former item is the input power from the driver of the motor, while the latter one is the variant quantity of the energy storage of the rotor. In order to eliminate the sampling error of the system, the multi-period average value is used, and $\mathrm{k}$ is the period number. After the load power is compensated by the feed-forward method, the control for the permanent magnet synchronous motor can always be equivalent to the no-load control, and the impact of the load uncertainty on the system is weakened. 


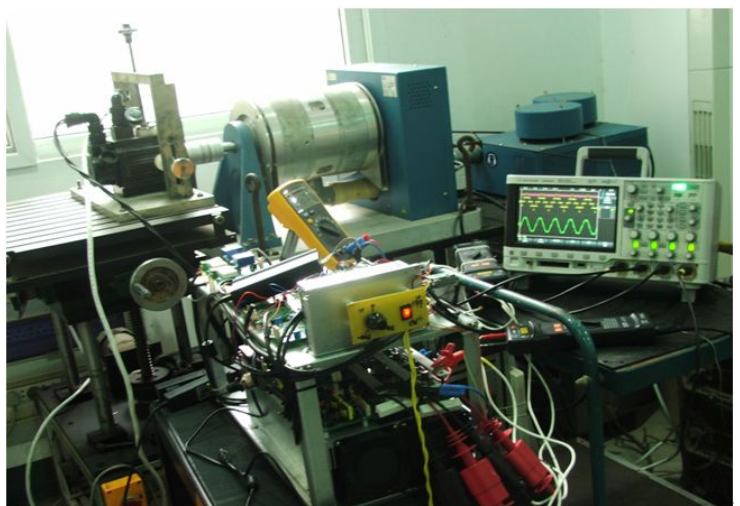

Fig. 5. The PMSM drive system.

TABLE I

PARAMETERS OF THE PMSM

\begin{tabular}{l|l}
\hline Rated power $(\mathrm{KW})$ & 2 \\
\hline Rated torque $(\mathrm{N} \cdot \mathrm{m})$ & 7.7 \\
\hline Rated speed $(\mathrm{rpm})$ & 2500 \\
\hline Rated current $(\mathrm{A})$ & 7.5 \\
\hline Rotational inertia $(\mathrm{kg} \cdot \mathrm{m} 2)$ & $1.53 \times 10-3(\mathrm{PMSM})$ \\
\hline Rotational inertia $(\mathrm{kg} \cdot \mathrm{m} 2)$ & $\begin{array}{l}1.343 \times 10-2 \quad(\text { adding } \\
\text { the dynamometer })\end{array}$ \\
\hline Torque coefficient $(\mathrm{N} \cdot \mathrm{m} / \mathrm{A})$ & 1.03 \\
\hline Voltage constant $(\mathrm{V} / \mathrm{rpm})$ & $66 \mathrm{~V} / 1000 \mathrm{rpm}$ \\
\hline Pole pairs & 4 \\
\hline
\end{tabular}

\section{RESUlts AND ANALYSIS OF THE EXPERIMENT}

In order to verify the feasibility of the vector control strategy based on energy feedback for PMSMs and its advantages over traditional vector control, a PMSM control system was set up in the lab, as shown in Fig. 5. The details of the PMSM technical parameters are listed as Table I.

\section{A. Comparison between the Traditional Double-loop Control Strategy and the Proposed Control Strategy}

Fig. 6 and Fig. 7 show the motor phase current, q-axis current and speed response curve from the staring of the motor up 300rpm, 1000rpm and 2000rpm under the traditional double-loop control strategy and the proposed control strategy.

The two control strategies adopt different outer regulators, but the same current inner loop regulator. From the comparison of two methods in terms of the response characteristics of the motor at start-up, it can be seen that:

(1) The two control strategies exhibit consistent acceleration performance when the motor speed error is larger, the output of the outer PI speed regulator is saturated, and the motor accelerates at its maximum current.

(2) When the motor speed approaches a given value, the

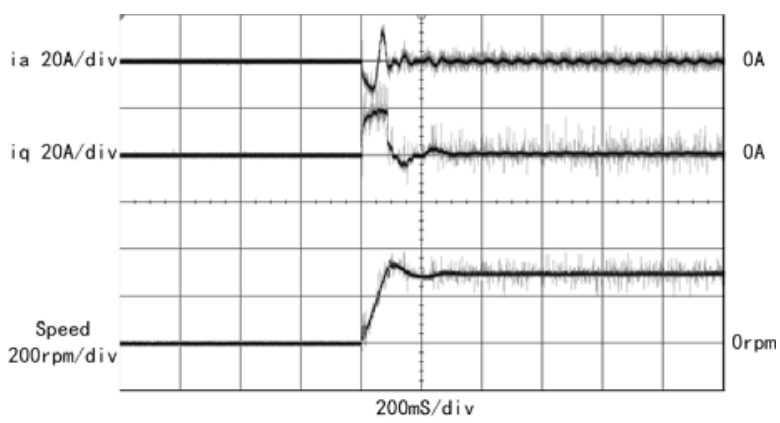

(a) $n_{\text {ref }}=300 \mathrm{rpm}$.

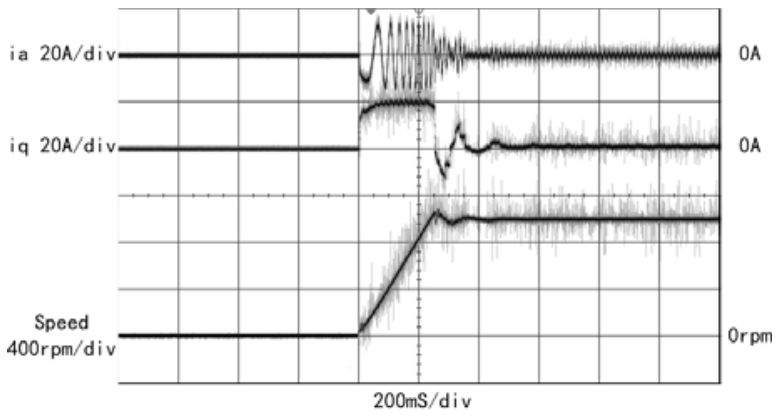

(b) $n_{\text {ref }}=1000 \mathrm{rpm}$.

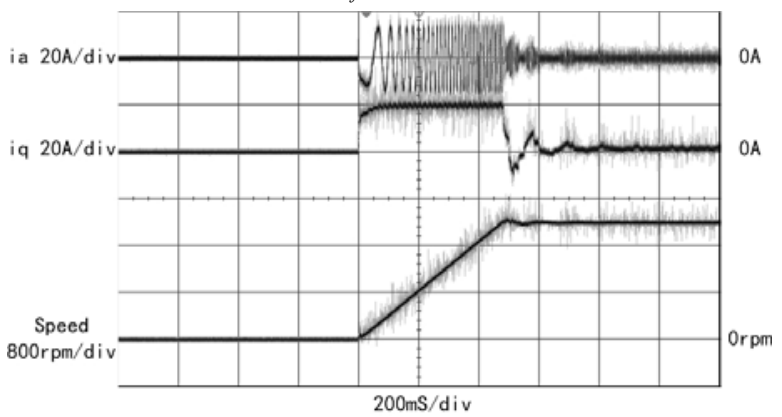

(c) $n_{\text {ref }}=2000 \mathrm{rpm}$.

Fig. 6. Experimental results using the traditional vector control.

P regulator of the outer loop PI regulator begins to decrease the control proportion, and the integrating regulator gradually increases the control proportion. By using the proposed control algorithm, the $\mathrm{P}$ regulator reduces the control output by the square law when the speed error is reduced. It can be seen that the control algorithm proposed in this paper is superior to the traditional double-loop control strategy in this process.

(3) When the motor speed is higher than a given value, the given current from the outer loop regulator will quickly drop to zero or negative. It can be seen that the control strategy proposed in this paper is superior to the traditional double-loop control strategy when the outer regulator desaturates.

In fact, the main difference between the two control strategies lies in the design of the outer loop. According to the q-axis current curve, when the motor speed is close to a given speed by the control strategy proposed in this paper, the q-axis current can be fast and stable and the speed oscillation 


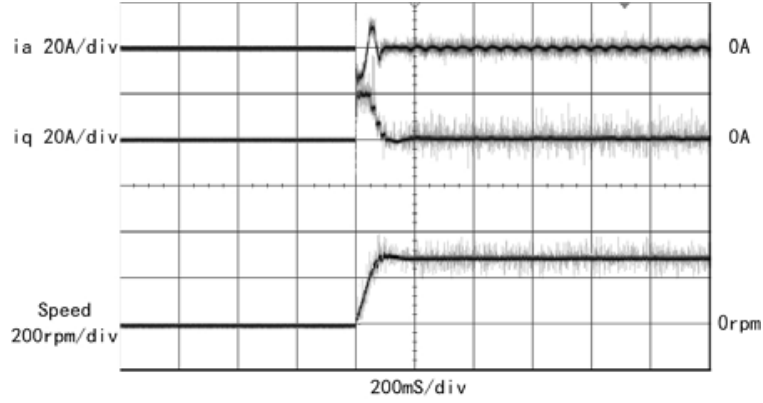

(a) $n_{\text {ref }}=300 \mathrm{rpm}$

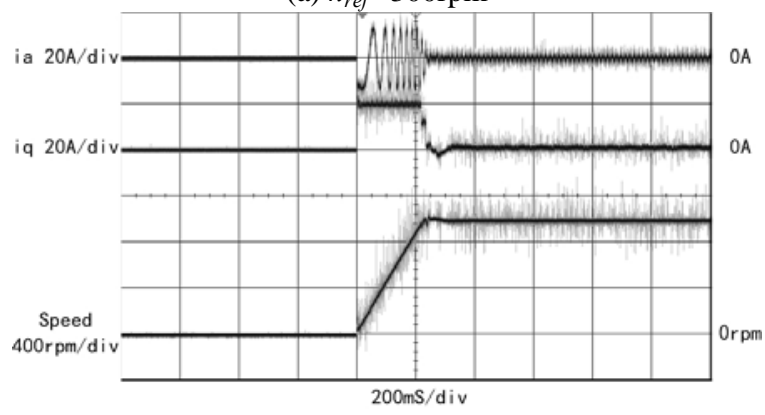

(b) $n_{r e f}=1000 \mathrm{rpm}$

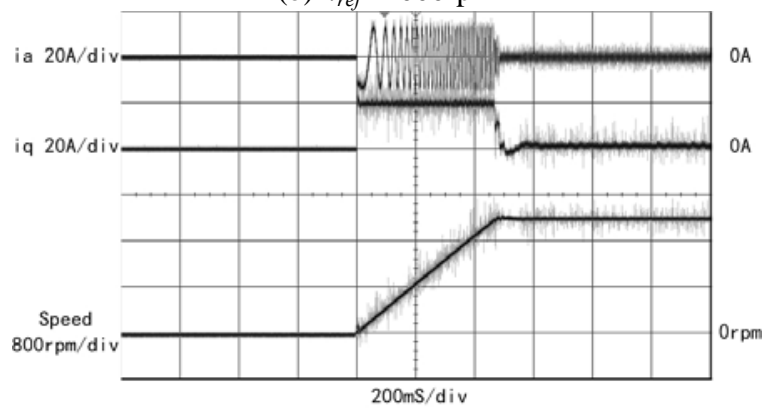

(c) $n_{\text {ref }}=2000 \mathrm{rpm}$

Fig. 7. Experimental results using vector control based on energy feedback.

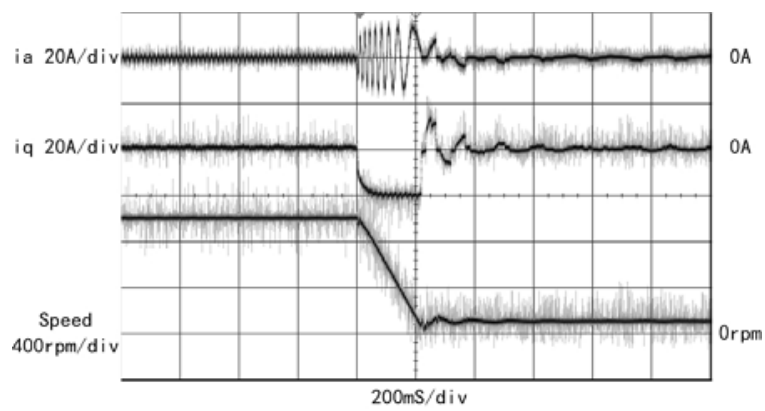

(a) Using the traditional vector control.

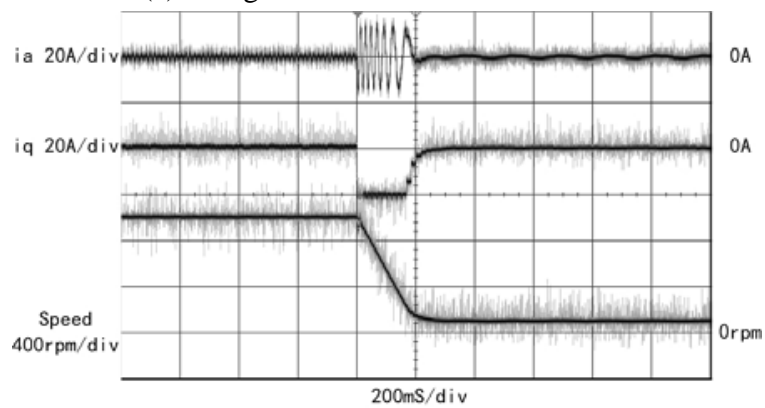

(b) Using vector control based on energy feedback.

Fig. 8. Experimental results of the deceleration process.

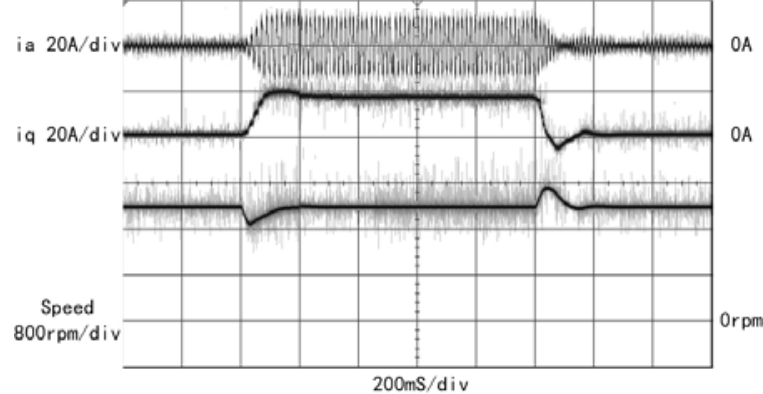

(a) Traditional vector control without load estimation.

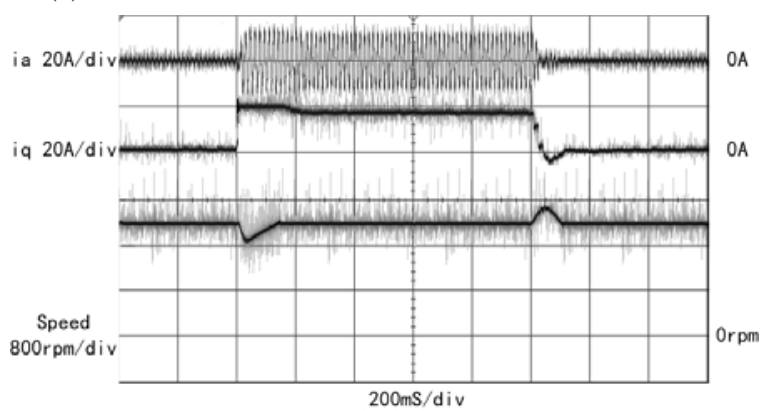

(b) The proposed control without load estimation.

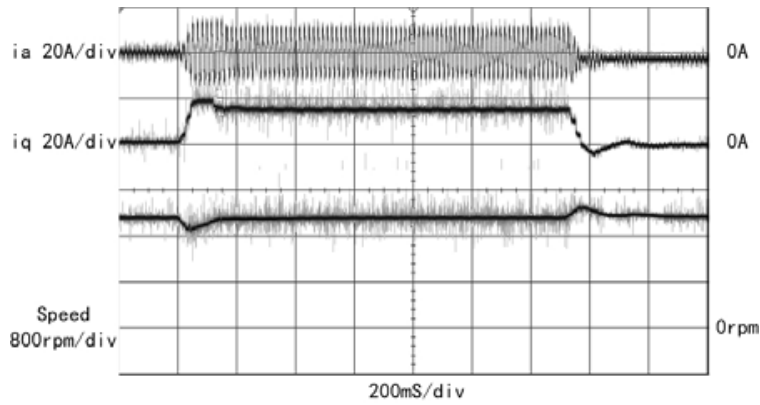

(c) Traditional vector control with load estimation.

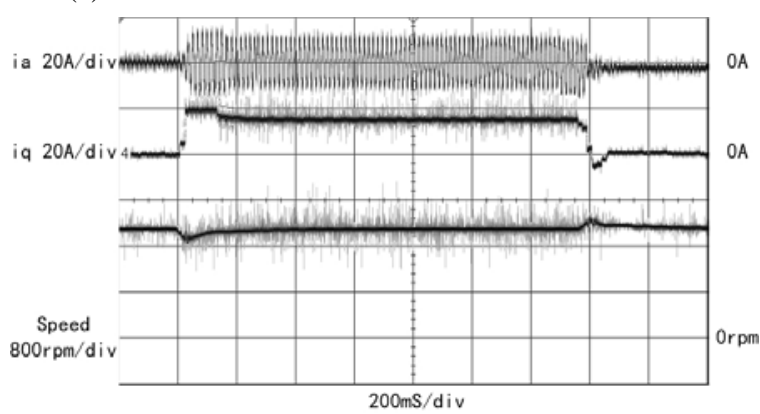

(d) The proposed control with load estimation.

Fig. 9. Experimental results in step load torque situation.

is effectively inhibited. A similar conclusion to the start-up process can also be drawn in the deceleration process, as shown in Fig. 8.

\section{B. Verification of the Algorithm with a Sudden Change of the Load Torque}

In order to verify the dynamic performance of the proposed algorithm, the traditional double-loop control strategy and the proposed control strategy are compared in terms of control performance by sudden changes of the load torque, and the experimental results are shown in Fig. 9. 


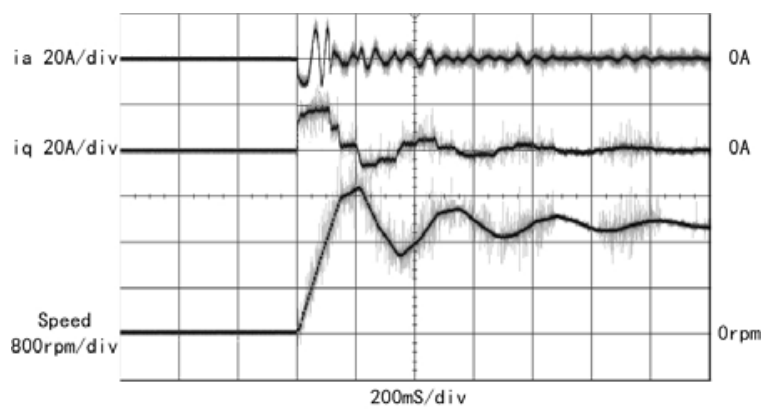

(a) Traditional vector control without load estimation.

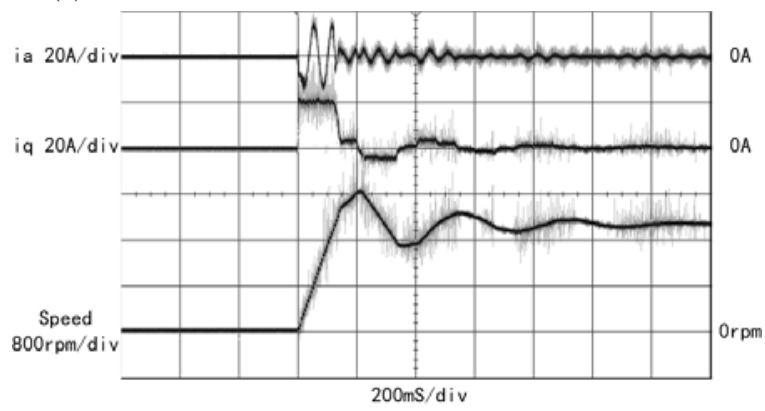

(b) The proposed control without load estimation.

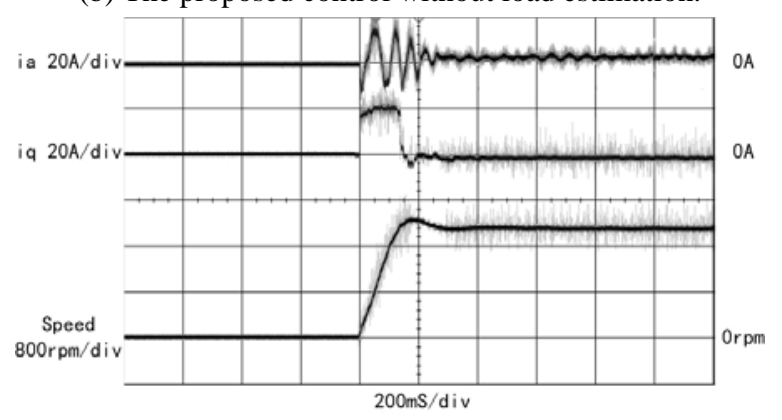

(c) Traditional vector control with load estimation.

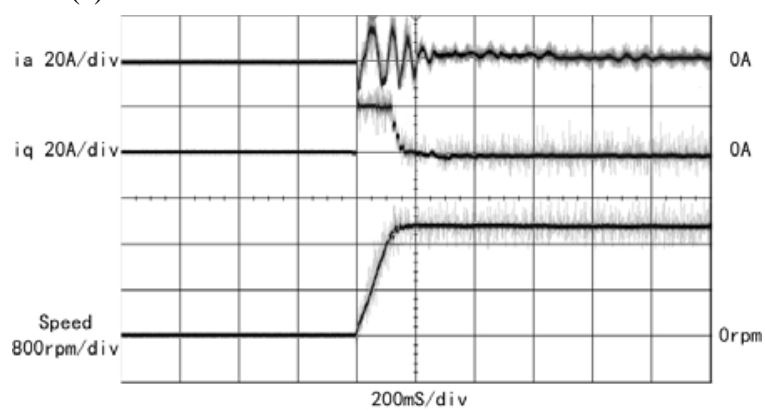

(d) The proposed control with load estimation.

Fig. 10. Experimental results in the load rotational inertia variation situation.

Fig. 9 (a) and (c) show the control performance without load estimation. As can be seen, when the load is increased suddenly, the speed drops significantly, and the given current from the outer loop is increased with a larger torque from the motor, resulting in an increased motor speed. As a result, the actual speed is restored to the given value. With the load estimation algorithm, the speed drop in the suddenly increased load and the speed rise in the suddenly decreased load are both superior. Due to the sudden change of the load torque, the stored kinetic energy of the motor and load system is absorbed. This results in a decreased motor speed. According to (23), the load power change can be estimated. The load estimation value is superimposed onto the given current of the outer loop. Thus, the feed-forward control to the power change is realized. In the four situations, the control performance of the proposed control strategy with load estimation is at its optimum.

\section{Verification of the Load Estimation Algorithm in the Load Rotational Inertia Variation Situation}

When the load machine is disengaged, the load rotational inertia changes to another value. Fig. 10 shows the response characteristics while re-starting the motor after the load inertia is changed under the traditional double-loop control strategy and the proposed strategy both with and without load estimation. As shown in Fig. 10 (a) and (b), when the rotational inertia of the load is greatly reduced, short-term oscillations appear in the two strategies without load estimation. In the actual system, the PI parameters of the outer loop must be modified to eliminate the oscillation. The start-up process is shown in fig. 10 (c) and (d), when the rotational inertia of the load is reduced greatly, the PI parameters remain as previously used in the above two experiments, and with the addition of load estimation. As can be seen, although the PI parameters are not changed, the two control strategies can achieve a response performance similar to that in Fig. 6 and Fig. 7. This indicates that the load estimation algorithm proposed in this paper is adaptable for changes of the load inertia.

\section{CONCLUSIONS}

From the perspective of energy storage and conversion, a vector control strategy based on the feedback of the rotor kinetic energy of a PMSM is proposed in this paper. Comparing the proposed strategy with the traditional vector control strategy, the following conclusions can be derived:

(1) The feasibility of the vector control strategy based on energy feedback is verified.

(2) The dynamic response of the vector control based on the rotor kinetic energy feedback of a PMSM is much better than that of traditional vector control, which has a smaller overshoot and response time.

(3) An effective load estimation method for the rotational inertia and torque variation of the load is presented which avoids complex estimation algorithms.

The proposed strategy can be applied to other control systems that require better rapidity as rectifiers and inverters.

\section{REFERENCES}

[1] T. Noguchi, H. Tomiki, and S. Kondo, "Direct power control of PWM converter without power-source voltage sensors," IEEE Trans. Ind. Appl., Vol. 34, No. 6, pp. 473-479, May/Jun. 1998. 
[2] J. B. Chu, Y. W. Hu, and W. X. Huang, "Basic theory of direct power control for PMSM," Trans. China Electrotech. Soc., Vol. 24, No. 10, pp. 21 -26, Oct. 2009.

[3] P. Cortes , M. P. Kazmierkowski , R. M. Kennel , D. E. Quevedo, and J. Rodriguez, "Predictive control in power electronics and drives," IEEE Trans. Ind. Electron., Vol. 55, No. 12, pp. 4312-4324, Dec. 2008.

[4] Y. Luo, Y. Q. Chen, H.S. Ahnc, and Y. G. Pi, "Fractional order periodic adaptive learning compensation for cogging effect in PMSM position servo system," American Control Conference, pp. 937-942, 2009.

[5] I. C. Baik, K.-H. Kim, and M. J. Youn, "Robust nonlinear speed control of PM synchronous motor using boundary layer integral sliding mode controltechnique," IEEE Trans. Control Syst. Technol., Vol. 8, No. 1, pp.47-54, Jan. 2000.

[6] S. H. Li, H. X. Liu. and S. H. Ding, "A speed control for a PMSM using finite-time feedback control and disturbance compensation," Tran. Inst. Measur. Contr., Vol. 32, No. 2, pp. 170-187, Feb.2010.

[7] H. X. Liu and S. H. Li, "Speed control for PMSM servo system using predictive functional control and extended state observer," IEEE Trans. Ind. Electron., Vol. 59, No. 2, pp.1171-1183, Feb.2012.

[8] F. F. M. El-Sousy, "Hybrid H-infinity-based wavelet-neural-network tracking control for permanent-magnet synchronous motor servo drives," IEEE Trans. Ind. Electron., Vol. 57, No. 9, pp.3157-3166, Sep. 2010.

[9] K. H. Kim and M. J. Youn, “A nonlinear speed control for a PM synchronous motor using a simple disturbance estimation technique," IEEE Trans. Ind. Electron., Vol. 49, No. 3, pp. 524 -535, Mar. 2002.

[10] Y. S. Kung and M. H. Tsai, "FPGA-based speed control IC for PMSM drive with adaptive fuzzy control," IEEE Trans. Power Electron., Vol. 22, No. 6, pp. 2476-2486, Jun. 2007.

[11] F. J. Lin and C. H. Lin, "A permanent magnet synchronous motor servo drives using self-constructing fuzzy neural network controller," IEEE Trans. Energy Convers., Vol. 19, No. 1, pp. 66-72, Mar.2003.

[12] S. H. Li and Z. G. Liu, "Adaptive speed control for permanent magnet synchronous motor system with variations of load inertia," IEEE Trans. Ind. Electron., Vol. 56, No. 8, pp.3050-3059, Aug. 2009.

[13] Y. A. R. I. Mohamed, "Design and implementation of a robust current control scheme for a PMSM vector drive with a simple adaptive disturbance observer," IEEE Trans. Ind. Electron., Vol. 54, No. 4, pp. 1981-1988, Apr. 2007.

[14] S. M. Yang and Y. J. Deng, "Observer-based inertia identification for autotuning servo motor drivers," 40th IAS Annu. Meeting, pp.968-972, 2005.

[15] H. H. Choi, N. T. T. Vu, and J. W. Jung, "Digital implementation of an adaptive speed regulator for a PMSM,” IEEE Trans. Power Electron., Vol. 26, No. 1, pp. 3-8, Jan. 2011.

[16] F. Hardan, J. A. M. Bleijs, R. Jones, and P. Bromley, "Bi-directional power control for flywheel energy storage system with vector-controlled induction machine drive," in Proc. Power Electronics and Variable Speed Drives, pp.477-482, 1998.

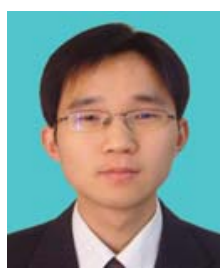

Anbang Wang was born in Anhui, China. He received his B.S. degree in Electrical Engineering from the Hefei University of Technology (HFUT), Hefei, Anhui, China, in 2002, where he is presently working toward his Ph.D. degree. His current research interests include power electronics, electric drives and diagnosis.

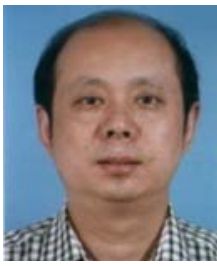

Qunjing Wang was born in Anhui, China. He received his B.S. degree in Electrical Engineering from the Hefei University of Technology (HFUT), Hefei, Anhui, China, in 1982, and his Ph.D. degree in Electrical Engineering from the University of Science and Technology of China, Hefei, Anhui, China. He is presently working as a Professor in the School of Electrical Engineering and Automation, HFUT. His current research interests include power electronics, electric drives and motor design.

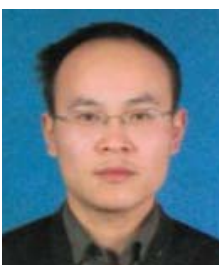

Weidong Jiang was born in Sichuan, China. He received his B.S. and Ph.D. degrees in Electrical Engineering from the Hefei University of Technology (HFUT), Hefei, Anhui, China, in 1999 and 2004, respectively. $\mathrm{He}$ is presently working as an Associate Professor in the School of Electrical Engineering and Automation, HFUT. His current research interests include power electronics, electric drives and power quality control. 